\title{
ADJOINT CLASSES OF LEBESGUE-STIELTJES INTEGRABLE FUNCTIONS
}

\author{
Abstract \\ This paper gives three pair of adjoint classes of the Lebesgue-Stieltjes \\ integrable functions.
}

\section{Introduction}

Let $a$ and $b$ be real numbers with $a<b$. Let $\mathcal{B}[a, b]$ be the class of all Borel measurable functions defined on $[a, b]$, and $\mathcal{F}[a, b]$ be the class of all realvalued functions defined on $[a, b]$. Let $g \in \mathcal{F}[a, b]$ and $g_{1}(x), g_{2}(x)$ be the positive, negative variations of $g$ over $[a, x]$ with $a \leq x \leq b$, respectively. If $g_{1}(x)+g_{2}(x)<\infty$ for any $x \in[a, b)$ and either $g_{1}(b)$ or $g_{2}(b)$ is finite, then we say $g \in E B V[a, b]$, the class of functions of extended bounded variation on $[a, b]$ (cf. [8]). If $g \in E B V[a, b]$, we have

$$
g(x)-g(a)=g_{1}(x)-g_{2}(x) \text { for any } x \in[a, b) .
$$

Since, for $i=1,2, g_{i}(x)$ is monotonically increasing on $[a, b)$, then there is a unique Baire measure $\mu_{g_{i}}$ such that

$$
\mu_{g_{i}}\left(a_{1}, b_{1}\right]=g_{i}\left(b_{1}+\right)-g_{i}\left(a_{1}+\right) \text { for all }\left[a_{1}, b_{1}\right] \subset[a, b]
$$

(define $\left.g_{i}(b+)=g_{i}(b)\right)$. Thus, in fact, a function $g \in E B V[a, b]$ gives rise to a $\sigma$-finite signed Baire measure $\mu_{g}=\mu_{g_{1}}-\mu_{g_{2}}$ on the class of all Borel sets in $[a, b]$ such that

$$
\mu_{g}\left(a_{1}, b_{2}\right]=g\left(b_{1}+\right)-g\left(a_{1}+\right) \text { for all }\left[a_{1}, b_{1}\right] \subset[a, b] .
$$

Key Words: Adjoint classes of functions, Lebesgue-Stieltjes integral, Baire measure, Borel sets and Borel measurable functions, and linear bounded functional

Mathematical Reviews subject classification: 26A42, 26A45, 28A25

Received by the editors October 21, 1999 
Now, for $f \in \mathcal{B}[a, b]$ and $g \in E B V[a, b]$, we define the Lebesgue-Stieltjes integral of $f$ with respect to $g$ by

$$
(\mathrm{L}-\mathrm{S}) \int_{a}^{b} f d g=\int_{a}^{b} f d \mu_{g}
$$

where $\mu_{g}$ is the $\sigma$-finite signed Baire measure called the Lebesgue-Stieltjes measure corresponding to $g$.

In the next section we shall use the definition in $[1,5]$ (only change the $(\mathrm{L}-\mathrm{S})$ integral for the (R-S) integral) to discuss the adjoint classes of the Lebesgue-Stieltjes integrable functions.

\section{Main Results}

In the present paper, besides the following classes of functions defined on $[a, b]$ :

- the class of functions of bounded variation $B V[a, b]$,

- the class of continuous functions of bounded variation $C B V[a, b]$, and

- the class of absolutely continuous functions $A C[a, b]$,

we shall also deal with the classes of functions as follows.

Definition 1. Let $g \in B V[a, b]$. Define $g^{*}(x)=g(x+)$ for $x \in[a, b)$ and $g^{*}(b)=g(b)$. If $g^{*} \in C B V[a, b](A C[a, b])$, then we say $g \in C_{o} B V[a, b]$ $\left(A C_{o}[a, b]\right)$.

Definition 2. A function $f \in \mathcal{B}[a, b]$ is said to belong to the class $B[a, b]$ if it is bounded on $[a, b]$.

Definition 3. A function $f \in \mathcal{B}[a, b]$ is said to belong to the class $B_{o}[a, b]$ if there is a number $N_{o}>0$ such that any closed subset of the set $E(x:|f(x)|>$ $N_{o}$ ) is at most countable.

In the following definitions we use $L^{p}[a, b](1 \leq p<\infty)$ to denote the space of all Lebesgue measurable functions $f$ on $[a, b]$ such that $(\mathrm{L}) \int_{a}^{b}|f|^{p}<\infty$, and use $L^{\infty}[a, b]$ to denote the space of all Lebesgue measurable functions on $[a, b]$ which are bounded except possibly a subset of Lebesgue measure zero.

Definition 4. Let $1 \leq q \leq \infty$. A function $f \in \mathcal{B}[a, b]$ is said to belong to the class $B^{q}[a, b]$ of $f \in L^{q}[a, b]$.

Definition 5. Let $1 \leq p \leq \infty$. A function $g \in \mathcal{F}[a, b]$ is said to belong to the class $A C_{o}^{p}[a, b]$ if $g \in A C_{o}[a, b]$ and $g^{\prime} \in L^{p}[a, b]$. 
Let $A$ and $B$ be two classes of functions defined on $[a, b]$. If $A$ and $B$ are adjoint with respect to the Lebesgue-Stieltjes integral, then it will be denoted by $A * B(L-S)$. We will prove the following theorems in the next section.

Theorem 1. $B[a, b] * B V[a, b](L-S)$.

Theorem 2. Let $1 / p+1 / q=1,1 \leq p \leq \infty . B^{q}[a, b] * A C_{o}^{p}[a, b](L-S)$.

Theorem 3. $B_{o}[a, b] * C_{o} B V[a, b](L-S)$.

\section{Proof of the Theorems}

Proof of Theorem 1.

(1) Suppose $f \in B[a, b]$ and $g \in B V[a, b]$. Let $\mu_{g}$ be the LebesgueStieltjes measure corresponding to $g$. The condition $g \in B V[a, b]$ implies that $\left|\mu_{g}\right|$ is a finite measure on $[a, b]$, and so $f$ is $\mu_{g}$-integrable on $[a, b]$. Thus, $(\mathrm{L}-\mathrm{S}) \int_{a}^{b} f d g=\int_{a}^{b} f d \mu_{g}$ exists.

(2) Suppose $g \in E B V[a, b]$ and $(\mathrm{L}-\mathrm{S}) \int_{a}^{b} f d g$ exists for all $f \in B[a, b]$. By the Hahn Decomposition Theorem ([7, p. 273]), there is a function $f \in B[a, b]$ with $|f| \leq 1$ such that

$$
\int_{a}^{b} f d \mu_{g}=\left|\mu_{g}\right|[a, b]
$$

Hence $\left|\mu_{g}\right|$ is a finite measure on $[a, b]$. That is, $g \in B V[a, b]$.

(3) Suppose $f \in \mathcal{B}[a, b]$ and $(\mathrm{L}-\mathrm{S}) \int_{a}^{b} f d g$ exists for all $g \in B V[a, b]$. Claim that $f \in B[a, b]$. Suppose $f \notin B[a, b]$. Then, there exists a sequence $\left\{a_{n}\right\} \subset$ $[a, b]$ such that $a_{n}$ monotonically converges to a point $c \in[a, b]$, and $\left|f\left(a_{n}\right)\right| \uparrow \infty$ as $n \rightarrow \infty$. Without loss of generality, we may assume $a_{n} \uparrow c$ with $a_{0}=a$, $f(a)>0$, and $f\left(a_{n}\right)+\uparrow \infty$ as $n \rightarrow \infty$. Set

$$
b_{n}=f\left(a_{n}\right)+; \quad d_{n}=1 / b_{n}-1 / b_{n+1} \quad \text { and } \quad D_{-1}=0, \quad D_{n}=\sum_{0}^{n} d_{i} .
$$

Then, define $g(x)=D_{n-1}$ for each $n \geq 0$ if $x \in\left[a_{n}, a_{n+1}\right)$ and $g(x)=\lim D_{n}$ if $x \in[c, b]$. Since $\sum_{0}^{\infty} d_{i}<\infty$, so $g \in B V[a, b]$. But, since

$$
(\mathrm{L}-\mathrm{S}) \int_{a}^{b}(f+) d g=\sum_{0}^{\infty}\left(f\left(a_{i}\right)+\right) \mu_{g}\left(\left\{a_{i}\right\}\right)=\sum_{0}^{\infty} b_{i+1} d_{i}=\infty,
$$

the integral $(\mathrm{L}-\mathrm{S}) \int_{a}^{b} f d g$ does not exist, a contradiction. Consequently, $f \in$ $B[a, b]$. 
Proof of Theorem 2.

(1) Suppose $f \in B^{q}[a, b], g \in A C_{o}^{p}[a, b]$ with $1 / p+1 / q=1$. Since

$$
(\mathrm{L}-\mathrm{S}) \int_{a}^{b} f d g=(\mathrm{L}-\mathrm{S}) \int_{a}^{b} f d g^{*}=(\mathrm{L}) \int_{a}^{b} f g^{\prime} d x
$$

and so the fact that $f \in B^{q}[a, b]$ and $g^{\prime} \in L^{p}[a, b]$ implies (L-S) $\int_{a}^{b} f d g$ exists.

(2) Let $1 \leq p<\infty$. Suppose $f \in \mathcal{B}[a, b]$ and $(\mathrm{L}-\mathrm{S}) \int_{a}^{b} f d g$ exists for all $g \in$ $A C_{o}^{p}[a, b]$. Whence, (L) $\int_{a}^{b} f h d x$ exists for all $h \in L^{p}[a, b]$. Set $f_{n}(x)=f(x)$ if $|f(x)| \leq n$ and $f_{n}(x)=0$ otherwise. Now, for each $f_{n}, n=1,2, \ldots$, define a linear functional:

$$
F_{n}(h)=(\mathrm{L}) \int_{a}^{b} f_{n} h d x, \quad h \in L^{p}[a, b] .
$$

From the Hölder Inequality, it follows that $F_{n}$ is a bounded functional. Since $\left|f_{n} h\right| \leq|f h|$ and $f h \in L[a, b]$, we have that

$$
\lim F_{n}(h)=(\mathrm{L}) \int_{a}^{b} f h d x, \quad h \in L^{p}[a, b]
$$

by the Lebesgue Convergence Theorem. By the Banach-Steinhaus Theorem ([3, p. 100]), $F(h)=\lim F_{n}(h)$ is a linear functional on $L^{p}[a, b]$. On the other hand, since

$$
L^{p}[a, b]^{*}=L^{q}[a, b] \text { with } 1 / p+1 / q=1 \text { and } 1 \leq p<\infty,
$$

where we denote the dual space of $A$ by $A^{*}$, there exists a unique function $f_{1} \in L^{q}[a, b]$ such that

$$
F(h)=(\mathrm{L}) \int_{a}^{b} f_{1} h d x, \quad h \in L^{p}[a, b] .
$$

So, we have

$$
\text { (L) } \int_{a}^{b}\left(f-f_{1}\right) h d x=0 \quad \text { for all } h \in L^{p}[a, b] .
$$

Set $h=\chi[a, t] \in L^{p}[a, b]$. Then

$$
\text { (L) } \int_{a}^{t}\left(f-f_{1}\right) d x=0 \text { for } t \in[a, b] \text {. }
$$


Thus, $f=f_{1}$ almost everywhere, and so $f \in L^{q}[a, b]$. Hence, $f \in B^{q}[a, b]$. Let $p=\infty$. If set $g \equiv x \in A C_{o}^{\infty}[a, b]$, then the fact that $(\mathrm{L}-\mathrm{S}) \int_{a}^{b} f d g=$ (L) $\int_{a}^{b} f d x$ exists implies $f \in L^{1}[a, b]$. Hence, $f \in B^{1}[a, b]$.

(3) Let $g \in E B V[a, b]$. Suppose (L-S) $\int_{a}^{b} f d g$ exists for all $f \in B^{q}[a, b]$, $1 \leq q \leq \infty$. We shall prove $g \in A C_{o}^{p}[a, b]$ with $1 / p+1 / q=1$. First of all, we are going to show it in the case $q=\infty(p=1)$. In order to prove $g \in A C_{o}[a, b]$, it suffices to prove that $\left|\mu_{g}\right|(E)=0$ for any Borel set $E \subset[a, b]$ with $m(E)=0$. By the Hahn Decomposition Theorem, we can define a function $f \in B^{\infty}[a, b]$ such that

$$
(\mathrm{L}-\mathrm{S}) \int_{a}^{b} f d g=(\mathrm{L}-\mathrm{S}) \int_{E} f d g=+\infty \cdot\left|\mu_{g}\right|(E)<\infty .
$$

This means $\left|\mu_{g}\right|(E)=0$, and so $g \in A C_{o}[a, b]$. Secondly, we are going to show $g \in A C_{o}^{p}[a, b]$ for $1 \leq q<\infty$ with $1 / p+1 / q=1$. From the preceding proof for the case $q=\infty$ and $B^{\infty}[a, b] \subset B^{q}[a, b]$, it follows that $g \in A C_{o}[a, b]$. So,

$$
\text { (L) } \int_{a}^{b} f g^{\prime} d x=(\mathrm{L}-\mathrm{S}) \int_{a}^{b} f d g^{*}=(\mathrm{L}-\mathrm{S}) \int_{a}^{b} f d g
$$

exists for all $f \in B^{q}[a, b], 1 \leq q<\infty$. Hence, we can define a linear functional

$$
F(f)=(\mathrm{L}) \int_{a}^{b} f g^{\prime} d x, \quad f \in B^{q}[a, b] .
$$

Since $B^{q}[a, b]$ is dense in $L^{q}[a, b]$, and so it follows from the proof in (2) that $g^{\prime} \in L^{p}[a, b]$ with $1 / p+1 / q=1$, thus $g \in A C_{o}^{p}[a, b]$.

Proof of Theorem 3.

(1) Let $f \in B_{o}[a, b]$ and $g \in C_{o} B V[a, b]$. Suppose any closed subset of the set $E\left(x:|f|>N_{o}\right)$ is countable. Since the Lebesgue-Stieltjes measure $\mu_{g}$ is regular, there exists a sequence $\left\{P_{n}\right\}$ of closed sets such that $P_{n} \subseteq E(x$ : $|f|>N_{o}$ ) for all $n \geq 1$ and

$$
\left|\mu_{g}\right|\left(P_{n}\right) \rightarrow\left|\mu_{g}\right| E\left(x:|f|>N_{o}\right) \text { as } n \rightarrow \infty .
$$

Since $g \in C_{o} B V[a, b]$ and $P_{n}$ is countable, and so $\left|\mu_{g}\right|\left(P_{n}\right)=0$ for all $n \geq 1$. Hence, it follows that $\left|\mu_{g}\right| E\left(x:|f|>N_{o}\right)=0$. Consequently, the integral $(\mathrm{L}-\mathrm{S}) \int_{a}^{b} f d g$ exists.

(2) Suppose $g \in E B V[a, b]$ and $(\mathrm{L}-\mathrm{S}) \int_{a}^{b} f d g$ exists for all $f \in B_{o}[a, b]$. Since $B[a, b] \subset B_{o}[a, b]$, and so $g \in B V[a, b]$ by Theorem A. Let $c \in[a, b]$. 
Define a function $f$ as follows: $f(x)=\infty$ if $x=c$, and 0 if $x \in[a, b] \sim\{c\}$. It is obvious that $f \in B_{o}[a, b]$. By hypothesis, the integral

$$
(\mathrm{L}-\mathrm{S}) \int_{a}^{b} f d g=(\mathrm{L}-\mathrm{S}) \int_{\{c\}} f d g=f(c) \mu_{g}\{c\}
$$

is finite. But, since $f(c)=\infty$, this implies $g^{*}(c)-g^{*}(c-)=\mu_{g}\{c\}=0$. Hence, $g^{*}(x)$ is continuous at $x=c$. Therefore, $g \in C_{o} B V[a, b]$.

(3) Suppose $f \in \mathcal{B}[a, b]$ and (L-S) $\int_{a}^{b} f d g$ exists for all $g \in C_{o} B V[a, b]$. We claim $f \in B_{o}[a, b]$. If $f \notin B_{o}[a, b]$, then for any $N>0$ the set $E(x:|f|>N)$ contains a closed subset, which is uncountable and so must contain a perfect subset ([6, p. 130]). Hence, we construct a function $g \in C_{o} B V[a, b]$ such that the integral $(\mathrm{L}-\mathrm{S}) \int_{a}^{b} f d g$ does not exist. First of all, since $A C_{o}[a, b] \subset$ $C_{o} B V[a, b]$, so $f \in B^{\infty}[a, b]$ by Theorem B. Thus, there exists a number $N_{o}>0$ such that for each $n>N_{o}$ the set $E(x:|f|>n)$ contains a Cantor set $S_{n}$ with $m\left(S_{n}\right)=0$. Set $x_{n}=\max \left(S_{n}\right)$ for each $n>N_{o}$. If necessary, we can modify those Cantor sets so that $x_{n} \neq x_{m}$ if $n \neq m$. Let $\eta$ be a cluster point of the sequence $\left\{x_{n}\right\}$. Without loss of generality we may assume there exists a subsequence $\left\{x_{n_{k}}\right\}$ of $\left\{x_{n}\right\}$ such that $x_{n_{k}} \uparrow \eta(k \rightarrow \infty)$. Now, we construct a function $g$ as follows. For $k=1$, let $y_{n_{1}}=\min \left(S_{n_{1}}\right)$. In the same way as in the proof of Theorem 2.2 in [2] we define $g(x)$ as a Cantor function on $\left[y_{n_{1}}, x_{n_{1}}\right]$, which is locally constant on $\left[y_{n_{1}}, x_{n_{1}}\right] \sim S_{n_{1}}$ with the range $\left[0,1-1 / n_{1}\right]$, and $g(x)=0$, if $x \in\left[a, y_{n_{1}}\right)$. In general, for each $k>1$ we define $g(x)$ as follows. Noting that $S_{n_{k}} \cap\left[x_{n_{k-1}}, x_{n_{k}}\right]$ is also a Cantor set with measure zero, let $y_{n_{k}}=\min \left(S_{n_{k}} \cap\left[x_{n_{k-1}}, x_{n_{k}}\right]\right)$ and define $g(x)$ as a Cantor function on $\left[y_{n_{k}}, x_{n_{k}}\right]$, which is locally constant on $\left[y_{n_{k}}, x_{n_{k}}\right] \sim S_{n_{k}}$ with the range $\left[1-1 / n_{k-1}, 1-1 / n_{k}\right]$, and $g(x)=1-1 / n_{k-1}$, if $x \in\left[x_{n_{k-1}}, y_{n_{k}}\right)$. Obviously, through this way we can define $g(x)$ for any $x \in[a, \eta)$. If we define $g(x)=1$ on $[\eta, b]$, we have $g \in C_{o} B V[a, b]$. Since $|f(x)|>n_{k}$ for $x \in S_{n_{k}} \cap\left[x_{n_{k-1}}, x_{n_{k}}\right]$ and

$$
\mu_{g}\left(S_{n_{k}} \cap\left[x_{n_{k-1}}, x_{n_{k}}\right]\right)=1 / n_{k-1}-1 / n_{k},
$$

we have

$$
\begin{gathered}
(\mathrm{L}-\mathrm{S}) \int_{a}^{b}|f(x)| d g \geq(\mathrm{L}-\mathrm{S}) \int_{x_{n_{1}}}^{\eta}|f(x)| d g \geq \sum_{k=2}^{\infty}(\mathrm{L}-\mathrm{S}) \int_{x_{n_{k-1}}}^{x_{n_{k}}}|f(x)| d g \\
\geq \sum_{k=2}^{\infty}(\mathrm{L}-\mathrm{S}) \int_{S_{n_{k}} \cap\left[x_{n_{k-1}}, x_{n_{k}}\right]}|f(x)| d g \geq \sum_{k=2}^{\infty} n_{k}\left(1 / n_{k-1}-1 / n_{k}\right) \\
=\sum_{k=2}^{\infty}\left(n_{k}-n_{k-1}\right) / n_{k-1}=\infty .
\end{gathered}
$$


Consequently, the integral (L-S) $\int_{a}^{b}|f| d g$ does not exist, and neither does the integral $(\mathrm{L}-\mathrm{S}) \int_{a}^{b} f d g$. But, this contradicts the hypothesis, hence we must have $f \in B_{o}[a, b]$.

\section{References}

[1] H. Chen, A pair of adjoint classes of Riemann-Stieltjes integrable functions, Real Analysis Exchange 23(1997/8), No. 1, 235-240.

[2] H. Chen, Adjoint classes of generalized-Stieltjes integrable functions, Real Analysis Exchange 24(1998/9), No. 1, 139-148.

[3] J. B. Conway, A Course in Functional Analysis, Springer-Verlag, New York, 1985.

[4] B. R. Gelbaum and J. M. H. Olmsted, Counterexamples in Analysis, Holden-Day, San Francisco, 1964.

[5] T. H. Hildebrandt, Introduction to the Theory of Integration, Academic Press, New York and London, 1963.

[6] E. Kamke, Theory of sets, Dover publications, New York, 1950.

[7] H. L. Royden, Real Analysis, 3 ed., Macmillan, New York, London, 1988.

[8] S. Saks, Theory of the integral, Dover, New York, 1964. 
